\title{
Exploring the Teaching Efficiency of Integrating an Animated Agent into Web-Based Multimedia Learning System
}

\author{
Kai-Yi CHIN $^{\dagger}$, Nonmember, Yen-Lin $\mathrm{CHEN}^{\dagger \dagger}$, Member, Jong-Shin $\mathrm{CHEN}^{\dagger \dagger \dagger}$, Zeng-Wei HONG ${ }^{\dagger \dagger \dagger \dagger a)}$, \\ and Jim-Min LIN ${ }^{\dagger}$, Nonmembers
}

\begin{abstract}
SUMMARY In our previous project, an XML-based authoring tool was provided for teachers to script multimedia teaching material with animated agents, and a stand-alone learning system was designed for students to display the material and interact with animated agents. We also provided evidence that the authoring tool and learning system in computer-assisted learning systems successfully enhances learning performance. The aim of this study is to continue the previous project, to develop a Web-based multimedia learning system that presents materials and an animated agent on a Web browser. The Web-based multimedia learning system can provide an opportunity for students to engage in independent learning or review of school course work. In order to demonstrate the efficiency of this learning system, it was applied to one elementary school. An experimental material, 'Road Traffic Safety', was presented in two learning systems: a Web-based PowerPoint learning system and a Web-based multimedia learning system. The experiment was carried out in two classes that had a total of thirtyone 3rd-grade students. The results suggest that using our authoring tool in a Web-based learning system can improve learning, and in particular, enhance learners' problem-solving ability. Students with higher achievement on the post-test showed better comprehension in problem-solving questions. Furthermore, the feedback from the questionnaire surveys show students' learning interest can be fostered when an animated agent is integrated into multimedia teaching materials, and that students prefer to adopt the Web-based multimedia learning system for independent learning after school.
\end{abstract}

key words: animated agents, Web-based learning environments, multimedia learning, interactive learning environment, e-learning

\section{Introduction}

A new technology emerges that incorporates animated agents into instruction for creating powerful multimedia learning environments. Animated agents are life-like characters on the computer screen with embody life-like behaviors, such as body gestures and facial expressions. Animated agents enable multimedia learning environments to combine verbal (spoken words) and non-verbal (e.g., emo-

\section{Manuscript received June 29, 2010.}

Manuscript revised October 22, 2010.

${ }^{\dagger}$ The authors are with the Department of Information Engineering and Computer Science, FengChia University, Taichung, 40724 Taiwan.

${ }^{\dagger}$ The author is with the Department of Computer Science and Information Engineering, National Taipei University of Technology, Taipei, 10608 Taiwan.

${ }^{\dagger \dagger}$ The author is with the Department of Information and Communication Engineering, Chaoyang University of Technology, Taichung, 41349 Taiwan.

${ }^{+t^{+\dagger}}$ The author is with the Department of Computer Science and Information Engineering, Asia University, Taichung, 41354 Taiwan.

a)E-mail: stewart@asia.edu.tw

DOI: 10.1587/transinf.E94.D.754 tion, gesture, motion) forms of communication; thus, they are capable of facilitating the students' engagement in the learning process [1], [2]. Empirical studies have demonstrated that learners have better results after interacting with learning systems which incorporate animated agents [3], [4]. For example, Qi Dunsworth and Robert K. Atkinson [2] designed an animated agent's image for the purpose of increasing a learner's attention. This agent can improve a learner's ability to understand the human cardiovascular system. Atkinson also made another study which adopted an animated agent, Peedy, to deliver instructions either textually or aurally [1]. The authors emphasized that learners can associate verbal information with Peedy's gestures and gaze.

W. Lewis Johnson and Jeff W. Rickel assert that a successful animated agent design must take into account the capabilities of the platform and network that will be used [5]. They also consider that integrating animated agents into Web-based learning materials is an interesting problem. Furthermore, research proposed the development of a Webbased learning system which designs animated agents as tutors or learning companions for their on-line courses [5]-[7]. Maomi Ueno [7] further found that animated agents can improve the interactivity of Web-based materials and enhance the learners' motivation. In recent years, most existing research emphasizes how to increase the animated agent's ability and intelligence to engage and motivate learners. However, teachers who are not animators or computer experts have difficultly learning to create a Web-based learning system with animated agents since they lack the experience or training; this is especially true for the majority of teachers in elementary education. They usually use an HTML editor (ex. Microsoft FrontPage) to create web pages or have had experiences of embedding PowerPoint slide shows in web pages. Thus, these teachers do not have the capability to create animated agents in Web-based instruction materials

In our previous project, we designed an authoring tool for scripting multimedia teaching material with animated agents [8]-[10]. This authoring tool provides teachers with a friendly virtual editor to help them edit instructional materials and script animated agents without complicated computer and animation knowledge, and also gives students a learning system which has to be installed on a PC to display their teachers' materials. The questionnaires completed by the teachers and pupils showed that the majority of the participants agreed that multimedia teaching material with animated agents is able to increase the pupils' motivation and 
engagement.

Therefore, the study presented here continues this project and develops a Web-based multimedia learning system that presents materials and an animated agent on the Web. Elementary students are able to interact with the designed Web-based multimedia learning system via a web connection and Internet browser. During this interaction, students are free to learn subjects from multimedia teaching materials and receive advice from animated agents without the boundary of the classroom. Thus, the Web-based multimedia learning system also provides an opportunity for students to engage in independent learning or review of school course work.

An evaluation was made between two groups of students who received either a course presented by the Webbased multimedia learning system, or the Web-based PowerPoint learning system. All of students' results were examined. We used a Web-based PowerPoint learning system because a lot of research proposes that PowerPoint is a presentation staple in computer assisted learning environments [11]. This research thinks that PowerPoint is a user-friendly application that can be used for the creation of visually clear, dynamic and attention-capturing presentations [12], [13]. Thus, PowerPoint is widely used in education around the world [11], [14]. The majority of teachers in Taiwan's elementary education have experience in using PowerPoint to produce Web-based instruction materials. PowerPoint slides can be linked by standard hyperlinks in web pages, and thus students can acquire the PowerPointbased materials via the Internet.

Our survey is primarily focused on the teaching efficacy of the Web-based multimedia learning system, and further explores students' attitudes toward the animated tutor and the two learning systems. Firstly, this paper investigates students' results after using the designed system. In the experiment, short-term memory is measured by using retention tests, and comprehension is measured by using problem-solving tests. This investigation shows that our Web-based multimedia learning system effectively teaches problem-solving skills in students. Secondly, the questionnaire was conducted to understand students' attitudes toward animated agents; the results show that integrating an animated agent into multimedia teaching materials efficiently fosters students' learning interest. In addition, the questionnaire also explores the overall significance of the students' attitudes towards the different instructional systems. The results show that the Web-based multimedia learning system satisfies more students than the Web-based PowerPoint learning system does.

The paper is organized as follows: in Sect. 2, we describe an overview of the existing multimedia curriculum script, and then explains the visual authoring tool. Section 3 introduces the Web-based multimedia learning system. In Sect. 4 , an overview of the evaluation method is given. Section 5 describes the results of the experiments we carried out. Finally, the last section presents our conclusions and future research direction.

\section{Multimedia Curriculum Script and the Authoring Tool}

Tsou and his colleagues [15] indicated that teachers have difficulties in integrating their instructional content into digital learning materials. If a teacher wants to customize a multimedia learning system, he/she will need the help of computer professionals. An appropriate solution is to provide a convenient authoring system. An authoring tool was developed in our previous study, which allows teachers to edit teaching materials that incorporate animated agents, as well as external multimedia objects, such as on-screen texts, images and video/audio files. The teaching material produced from this authoring tool, termed 'multimedia curriculum scripts,' is documented in XML tagging structures [8][10].

Referring to the segmenting principle [16], breaking a continuous lesson into bite-sized manageable segments can effectively reduce the cognition loads during the learner's essential cognitive process. Each segment conveys a maximum of three correlated elements. In other words, the teacher may try to combine multimedia from several sources, all pertaining to a common subject, together into a segment, and further link several segments together to make a lesson. Thus, the structure of a multimedia curriculum script in our design has three layers: the lesson layer, the segment layer and the tutor layer. Each script layer corresponds to an XML tagging structure.

\subsection{Tutor Script}

The animated tutors are constructed by using the Microsoft Agent animation engine. If the family of Microsoft Windows systems is installed, several default actors (i.e. Microsoft agents) are available, and each actor has a unique name. The speech function is able to specify a tutor's instructional advice in printed text and spoken voice. Based on modality principles [17], words (e.g. on-screen speech) in a learning system should be spoken rather than appear only as on-screen text. The Microsoft agent engine has a support function for an agent to vocalize its language, but this function is limited to English.

Figure 1 shows the example of a tutor script in our multimedia curriculum scripts. Three XML tags, 〈Actor $\langle$ Speech $\rangle$, and $\langle$ Motion $\rangle$, corresponding to the definition of a tutor, are used to script an animated agent. The 〈Reference〉 is an optional element which specifies the necessary external multimedia source files, such as movie clips, audio files, or other text files.

\subsection{Segment Script}

Figure 2 shows the example of a segment script. The XML tag, 〈Theme〉, is designed to specify the necessary multimedia assets of a segment. It allows a teacher to explain the segment material with images or video/audio files. The 


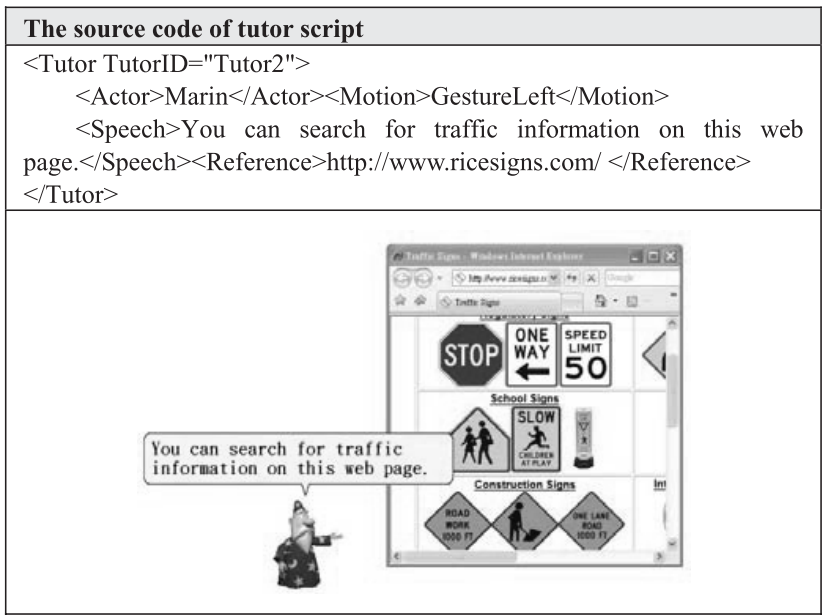

Fig. 1 An example of a simple tutor script.

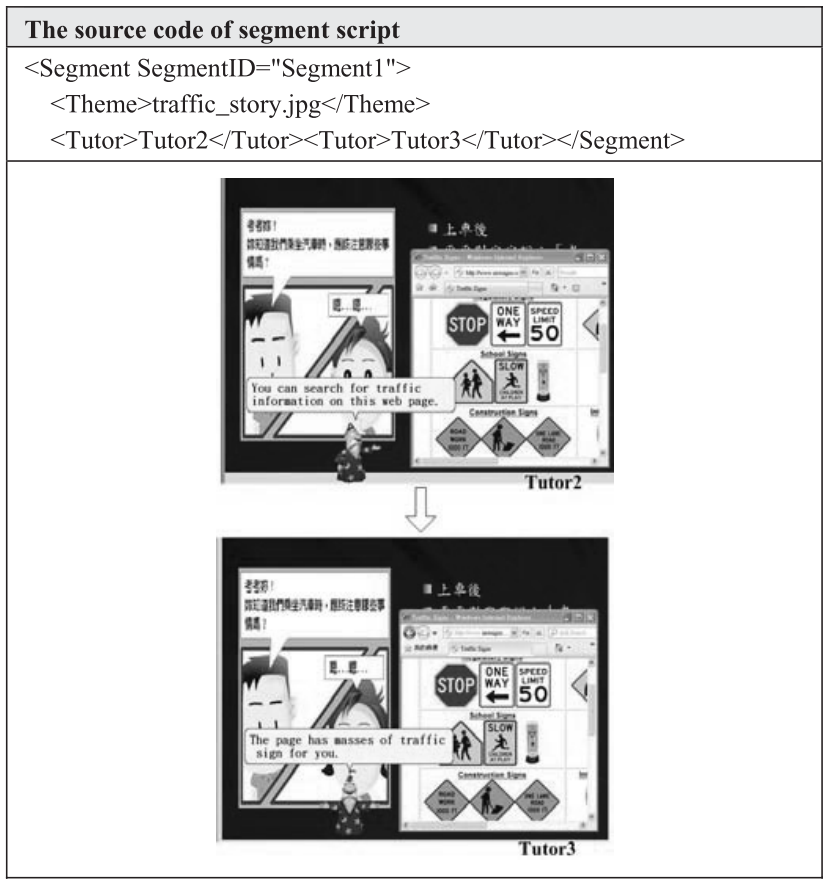

Fig. 2 An example of a simple segment script.

$\langle$ Tutor〉 is a tag to link to a tutor script. Therefore, it needs a sequence of $\langle$ Tutor $\rangle$ tags to specify the order in which the tutors appear. In Fig. 2, the $\langle$ Theme $\rangle$ element is used to specify a background picture to be the theme of this segment script. The segment script displays a sequence of two tutors scripted as Tutor 2 and Tutor 3. The segment contains two $\langle$ Tutor〉 tags in order to specify two tutor scripts.

\subsection{Lesson Script}

Makoto Takeya and his colleague assert that while a computer-assisted learning system provides a non-linear linking structure in the multimedia network, students are able to set up their own goals and create their own paths

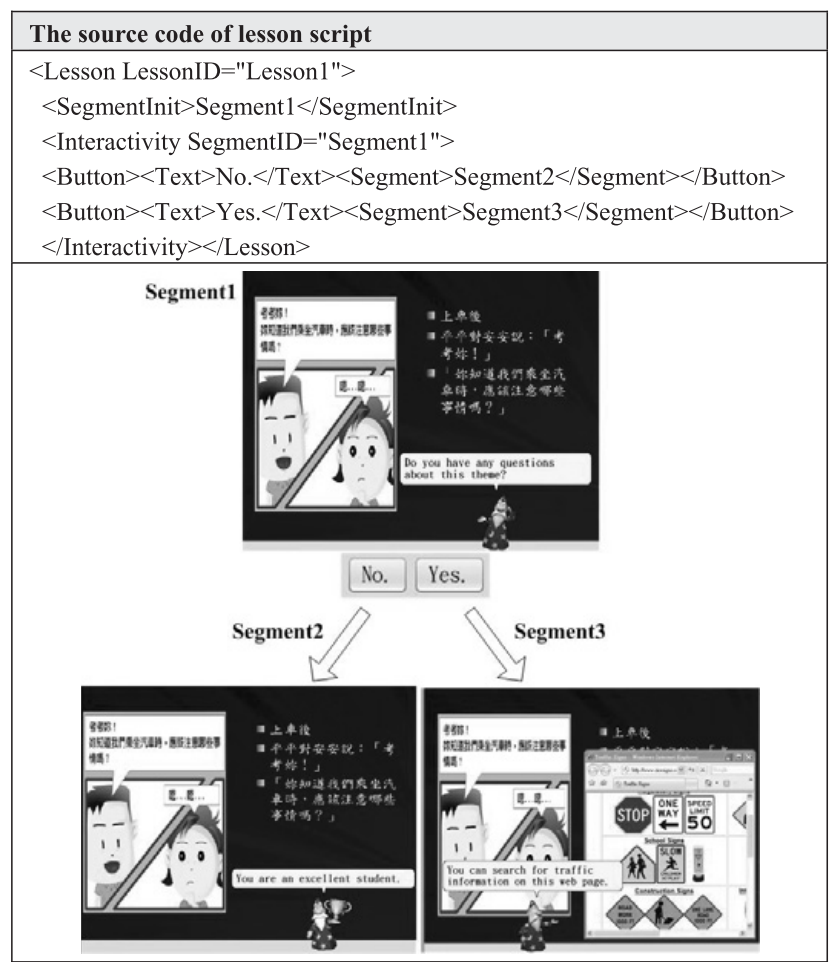

Fig. 3 An example of a simple lesson.

to help them engage in learning [18]. Other research further provides evidence that student navigation and interpretation of materials represented in non-linear content structure can enhance the effect of instruction in multimedia learning systems [18], [19]. Thus, in order to enhance adaptivity, the lesson script provides a non-linear interactive structure for students to decide their own learning directions based on their interests. Figure 3 shows an example of the lesson script. A mandatory tag, $\langle$ SegmentInit $\rangle$, is used to specify the starting segment in a lesson script. The 〈Interactivity tag has a SegmentID attribute for specifying a set of buttons to link to the following segments. The 〈Interactivity〉 tag also has a sub-tag, $\langle$ Button $\rangle$, for scripting the details of each button within $\langle$ Segment $\rangle$ and $\langle$ Text $\rangle$. The $\langle$ Segment $\rangle$ tag specifies which successive segment script will then be loaded, and the $\langle$ Text $\rangle$ tag specifies the caption for a button. In Fig. 3, the initial segment, Segment1, represents an animated tutor, and the lesson presents two buttons, "No." and "Yes.", respectively. The learner can click the 'No.' button to load the 'Segment2' segment, or click the 'Yes.' button to load the 'Segment3' segment.

\subsection{Authoring Tool}

The authoring tool provides a visual editor for a teacher to edit material contents pertaining to his/her subject by selecting and deploying the necessary multimedia objects (e.g., images, video/audio files, etc.). The teacher can also use the authoring tool to set up an animated agent by configuring its appearance and motion patterns, as well as its speech. The 
curriculum script generator of the authoring tool produces teaching material documented in three XML-based curriculum scripts. In other words, teachers can create, delete and modify a lesson script, including its segment scripts and tutor scripts, in an easy and intuitive manner using this authoring tool.

The current version of our authoring tool has been successfully developed for Microsoft Windows systems. The window-style layout of this visual editor helps teachers to become familiar with its manipulation. Figure 4 shows the user interface of the authoring tool. When a teacher creates a new curriculum by using the authoring tool, a lesson script will first be generated.

There are five main sections: Lesson Management, Theme Setting, Button Setting, Tutor Setting and Sequence Management. In the Lesson Management section, a window displays the current lesson structure in a diagram consisting of segments. Each segment is depicted as a rectangular block. The link between two segments is represented as a solid line. Moreover, Lesson Management provides a popup menu which comprises of two options: to insert a new segment, or to remove an existing segment from the current lesson structure. After adding or deleting segments, the lesson structure can be redisplayed immediately.

When one of the segments in the Lesson management section is selected, the Theme Setting section will provide a form for the teacher to edit the selected segment. The form provides GUI widgets that allow teachers to employ an existing multimedia file (e.g., images) to present the theme of the segment. Similarly, the Button Setting section provides GUI widgets for teachers to link the current segment to its successive segment by setting up a button. The Tutor Setting section provides a form for teachers to establish animated tutors in the current segment. The form allows for the adding or removing of a Microsoft agent in the current segment. It provides a set of GUI widgets to configure the appearance, speech and motion of an animated tutor, as well as the external multimedia files. Furthermore, the working sequence of an animated tutor is displayed in the Sequence Management section. Each line listed in the sequence corresponds to a tu-

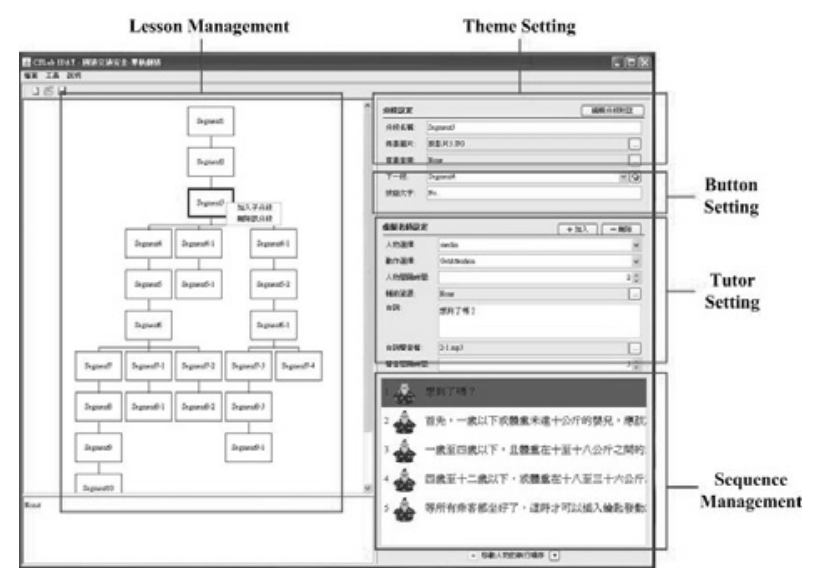

Fig. 4 Main page of the authoring tool. tor script. Thus, the teacher is able to decide and rearrange sequence by modifying the tutor scripts.

\section{Web-Based Multimedia Learning System}

The learning system provides web-page-based user interfaces for students to learn curriculum scripts authored by teachers. A student can read the learning content and interact with an animated agent via a Web browser (e.g., Microsoft Internet Explorer). A script interpreter is developed to interpret curriculum scripts and then present multimedia materials by invoking the corresponding multimedia object players, as well as present the animated agent by invoking the Microsoft Agent engine. There are two factors that influenced our decision to choose the Microsoft agent engine. Firstly, it takes a lot of time and effort to design programmable animated characters from scratch by using 2D/3D mpeg technology, which is unnecessary in Microsoft, whose agent has related supported APIs for programmers to specify the presence of a Microsoft agent. Secondly, Microsoft agents are cartoon-like avatars and each avatar has diverse default animated gestures and motions. Cartoon-like avatars make young pupils in elementary education more at ease and more entertained within the learning environment.

Figure 5 shows the main page of the learning system. It is divided into three sections: Exposition of the Curriculum Content, Control Panel and Tutor Dialogue. In the Exposition of the Curriculum Content section, there is a window to display the authored curriculum script. For example, Fig. 5 shows a curriculum script that presents an elementary education lesson: 'Road Traffic Safety'. An XML interpreter in the learning system is able to parse the lesson script and load the starting segment onto the displayed window. The multimedia object driver of the learning system then loads the corresponding graphics and video/audio narration that is specified in the segment script. The Microsoft agent driver of the learning system also loads the animated agent as spec-

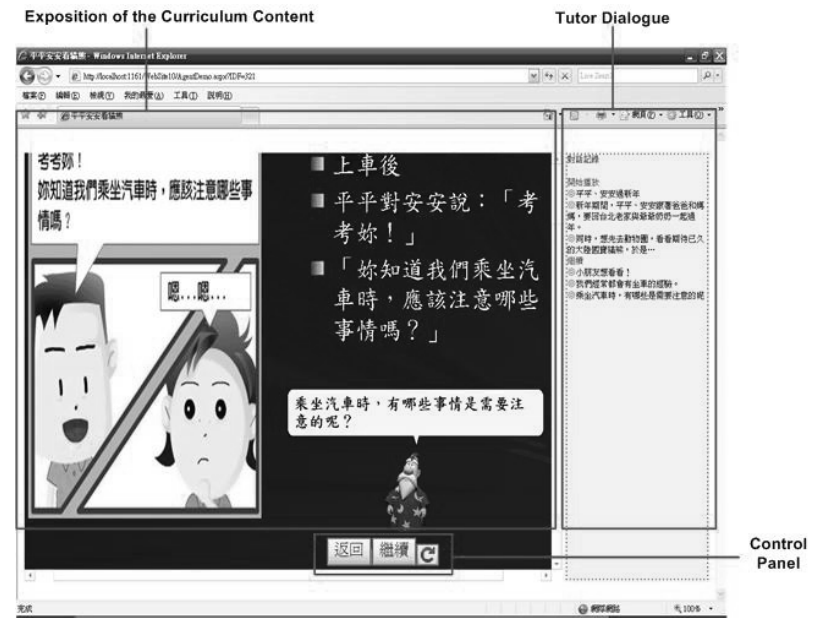

Fig. 5 The main page of the Web-based multimedia learning system. 
ified in the tutor script. Therefore, students can read the content on the screen and also receive visual/auditory advice from the animated agent.

The Control Panel section displays a set of buttons as specified by the teacher. In Fig. 5 example, the teacher edited three buttons using the authoring tool, a 'back' button, a 'next' button, and a 'replay' button, allowing the pupils to interact with the system by using buttons.

The Tutor Dialogue section is able to display the animated agent's previous dialogues on the right of the screen. This window thus allows students to review the animated agent's explanations and encourages them to recall the instructions.

\section{Evaluation Method}

\subsection{Materials and Apparatus}

The material for evaluation consists of thirty-four segments of a 'Road Traffic Safety' lesson. The content presents 'Taiwan Highway Traffic Safety' and was adapted from a 3rd-grade elementary textbook approved by the Taiwan's Ministry of Education. Thus, our evaluation did not use primary courses in Taiwan elementary education, such as 'mathematics' or 'science'. There are two factors that influenced our decision: firstly, the parents of the participating pupils did not want this evaluation to add to their child's study load; secondly, the comments from the participating teachers stated that a life education course would cause less pupil anxiety in the experiment.

Two teachers of the pupils in this experiment were invited to join the project. Both have worked in elementary education for many years. They also have experience and the basic skills for designing PowerPoint-based slides. Before designing and editing the materials in this experiment, both teachers were requested to participate in a tutorial introduced our authoring tool. As they started to edit the material for the experiment, they were requested to design a PowerPoint version. Then, the two teachers used the authoring tool to edit the Web-based multimedia learning version that corresponded with their Web-based PowerPoint version

Figure 6 shows the corresponding Web-based PowerPoint learning system. In each page, the pupils read the material presented on the screen. The navigation of the learning system, such as going back a single screen, replaying the current screen or moving to the next screen, is undertaken simply by the click of a mouse. In Fig. 6, when the pupils click their mouse on the screen, the learning system will advance the material content to the next page.

Figure 7 shows the corresponding Web-based multimedia learning system. This system combines images, the animated tutor's presentation, text explanations and instructions to advance the pupils' learning. Pupils can read the teaching content on the screen and accept visual/dynamic advice from the animated tutor. The animated tutor presents words and speaks corresponding to the modality and multimedia principles, and this facilitates a deeper level of under-

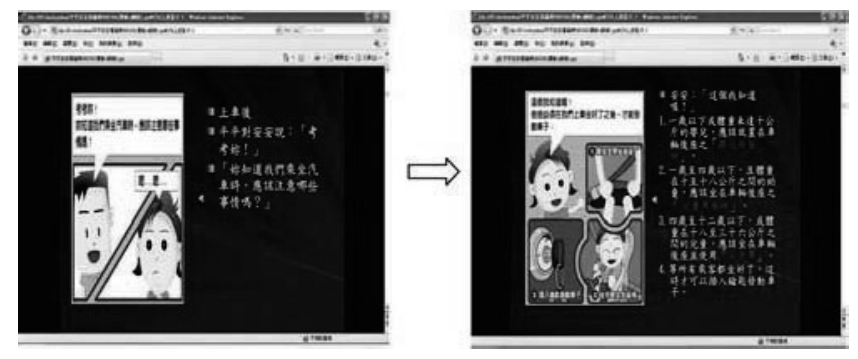

Fig. 6 Examples of the Web-based PowerPoint learning system.

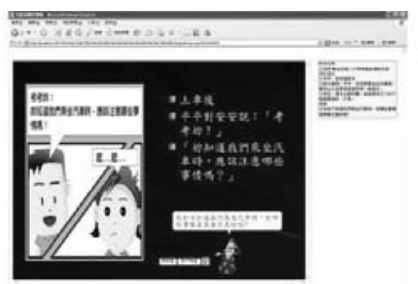

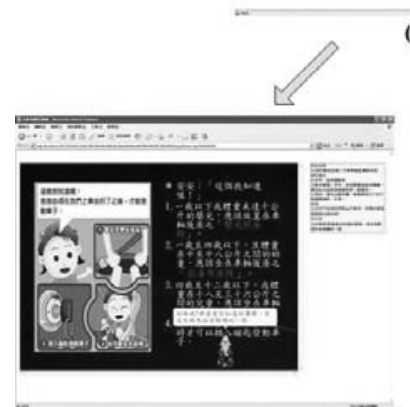

(b)

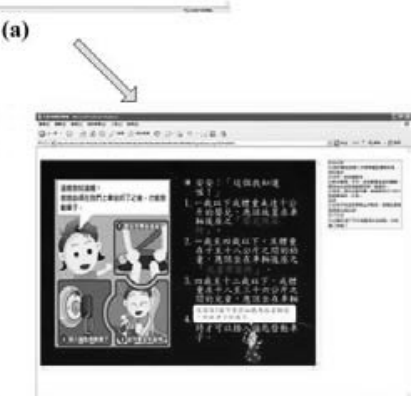

(c)
Fig. 7 Examples of the Web-based multimedia learning system.

standing in pupils through the associations made between verbal and visual presentations [17]. The dialogue window displays the animated tutor's previous dialogues on the far right of the screen.

In our experiment, the Web-based multimedia learning system not only provides a control panel for the pupils to set their own learning pace, but also enables the pupils to decide the animated tutor's actions. As shown in Fig. 7 (a), when the pupils press the left button, the learning system will change the material content from Fig. 7 (a) to Fig. 7 (b); when they press the middle button, the learning system will change the content to Fig. 7 (c). In other words, we use a non-linear interactive structure to enable students to decide animated tutors' advice and motion, and further to create an active relationship between the students and the learning system.

\subsection{Participants}

The participants are two classes of 31 3rd-grade pupils at the Chiau-Rung Elementary School in Taichung City, Taiwan. One class has 17 students and the other class has 14 students. 16 are male and 15 are female, and their ages range from 9 to 10 . The first class $(N=17)$ is assigned to use the Web-based 
PowerPoint learning system, and the other class $(N=14)$ is assigned to use the Web-based multimedia learning system. All participants are familiar with the basics of computers, such as mouse clicking and web-page browsing. Before the experiment, none of the students have viewed our materials.

A twenty-minute pre-test is given to all participants to measure their basic knowledge of the used material. The maximum total score of the pre-test is 100 . The pre-test means of the two groups are 52.94 and 51.76. The simple factorial $t$-test reveals no significant difference between the two groups; $t(29)=0.685, p>0.05$, indicates that the two groups are at the same level of knowledge with regard to the experimental materials.

\subsection{Procedure}

Each group enters one of two computer labs, which have been installed with Web-based multimedia learning systems and Web-based PowerPoint learning systems, respectively. All participating pupils are given one hour to complete the learning activities. When the participants are complete, everyone is given a twenty-minute post-test. After both groups have completed the post-test, the two groups are asked to switch computer labs. Thus, all participants experience the two systems. Finally, the participants fill out a questionnaire to evaluate their attitudes toward the animated tutor and the two learning systems.

\subsection{Scoring of the Post-Test}

All questions in the post-test were designed by the two teachers, who had designed the materials for the experiment. There are two types of questions: retention and problemsolving. The retention questions are twenty multiple-choice questions, in which students choose one of four answers. The multiple-choice questions are four points for each question answered correctly. Five problem-solving questions are used to measure students' comprehension and problemsolving ability. Each question is a statement which describes a traffic problem. Students have to underline the error and write the correct answer to complete each question. The problem-solving questions also are four points for each question answered correctly. The maximum total score of the post-test is 100 .

\subsection{Learning Attitudes}

A questionnaire of learning attitudes is conducted after finishing both systems. The first part of the questionnaire has six items which measure students' attitudes toward the animated agent's presentation. All items are measured on a five-point Likert scale in which 5 is "strongly agree" and 1 is "strongly disagree". The second part of the questionnaire consists of five items investigating which learning system the students prefer.

\section{Results}

The significant differences in the post-test between the two learning systems are assessed using simple factorial $t$-test analyses. Cohen's $d$ statistic is an effect size used to indicate the standardized difference between two means. It can be calculated as $d$ values of $0.2,0.5$ and 0.8 correspond to small, medium and large of the effect size, respectively [20]. All statistical analyses is performed with an alpha value of 0.05 .

\subsection{Post-Test Scores}

Table 1 presents the means and standard deviations for the overall, retention and problem-solving test scores in the post-test. In the overall post-test, the $t$-test reveals that the Web-based multimedia learning system has a significant difference in student performance, $t(27)=2.154, p<0.05$, and results of the post-test show that it $(M=69.29, \mathrm{SD}=$ 10.10 ) outperforms the Web-based PowerPoint learning system $(M=58.71, \mathrm{SD}=16.93)$. Cohen's $d$ statistic for this data yields an effect size estimate of 0.759 , which corresponds to a medium-to-large effect.

The retention results show that there are no significant differences in student performance between the two groups, $t(29)=0.808, p>0.05$, although the Web-based multimedia learning system achieved a higher mean score $(M=$ $49.71, \mathrm{SD}=10.25)$. The problem-solving $t$-test results reveal that the Web-based multimedia learning system has a significant difference in student performance, $t(17)=4.092$, $p<0.05$, and also show that the Web-based multimedia learning system $(M=19.57, \mathrm{SD}=1.16)$ outperforms the Web-based PowerPoint learning system $(M=12.59$, $\mathrm{SD}=6.92)$. Cohen's $d$ statistic for this data yields an effect size estimate of 1.407 , which corresponds to a large effect.

\subsection{Result of the Questionnaire}

From the data collected from the thirty-one students that have completed the learning activities on both instructional systems, the calculated Cronbach's alpha reliability for the questionnaire was 0.814 . This indicates that the collected data is highly reliable. Results of the post-questionnaire are listed in Tables 2 and 3.

The results show the general mean value of consensus

Table 1 Descriptive statistics for post-test scores.

\begin{tabular}{lcccccc}
\hline System & \multicolumn{2}{c}{ Overall } & \multicolumn{3}{c}{ Retention } & \multicolumn{2}{c}{ Problem-solving } \\
\cline { 2 - 7 } & Mean & SD & Mean & SD & Mean & SD \\
\hline $\begin{array}{l}\text { multimedia learning } \\
\text { system (n=14) }\end{array}$ & $69.29 *$ & 10.10 & 49.71 & 10.25 & $19.57^{*}$ & 1.16 \\
$\begin{array}{l}\text { PowerPoint learning } \\
\text { system (n=17) }\end{array}$ & 58.71 & 16.93 & 46.12 & 13.79 & 12.59 & 6.92 \\
\hline${ }^{*} p<0.05$ & & & & & & \\
\hline$p<0$
\end{tabular}


Table 2 The attitudinal survey to the animated agent's presentation.

\begin{tabular}{lll}
\hline & Mean & SD \\
\hline 1. You are satisfied with having the animated tutor in & 4.68 & 0.60 \\
$\begin{array}{l}\text { your learning. } \\
\text { 2. You pay attention to advice from the animated }\end{array}$ & 4.42 & 0.76 \\
$\begin{array}{l}\text { tutor. } \\
\text { 3. The animated tutor helps you to understand more }\end{array}$ & 4.61 & 0.80 \\
$\begin{array}{l}\text { about the teaching material. } \\
\text { 4. The animated tutor resembles a friend of yours and } \\
\quad \text { helps you to learn the multimedia materials. }\end{array}$ & 4.58 & 0.76 \\
$\begin{array}{l}\text { 5. You feel more relaxed while learning with the } \\
\text { animated tutor than with a human teacher. }\end{array}$ & 4.58 & 0.67 \\
$\begin{array}{l}\text { 6. You wish other multimedia materials also have } \\
\text { animated tutors. }\end{array}$ & 4.45 & 0.93 \\
\begin{tabular}{l} 
The overall average score. \\
\hline
\end{tabular} & 4.55 & 0.75 \\
\hline
\end{tabular}

Table 3 The preference survey for both learning systems.

\begin{tabular}{lll}
\hline Questions & \multicolumn{2}{l}{ Percentage of returns } \\
\cline { 3 - 3 } & $\begin{array}{l}\text { Web-based } \\
\text { multimedia } \\
\text { learning system }\end{array}$ & $\begin{array}{l}\text { Web-based } \\
\text { PowerPoint } \\
\text { learning system }\end{array}$ \\
\hline 1. I pay more attention when using & $90 \%$ & $10 \%$ \\
$\quad \begin{array}{l}\text { this system. } \\
\text { 2. This system is more interesting. }\end{array}$ & $94 \%$ & $6 \%$ \\
3. $\begin{array}{l}\text { This system increases my } \\
\text { motivation to continue learning. }\end{array}$ & $97 \%$ & $3 \%$ \\
4. This system helps me with & $90 \%$ & $10 \%$ \\
$\quad \begin{array}{l}\text { independent learning. } \\
\text { 5. I will use this system to review } \\
\text { school course work. }\end{array}$ & $94 \%$ & $6 \%$ \\
\hline
\end{tabular}

is 4.55 ( $\mathrm{SD}=0.75)$, indicating that the majority of participating pupils are "highly satisfied" with integrating animated agents in the proposed multimedia learning system. All mean values of the questions are higher than or equal to 4.00 , which means that the students hold affirmative opinions. In fact, existing studies found that an animated character can render a learning system more entertaining or engaging [3], [21], [22]. Virtual characters make learning systems more life-like, and this increases students' attention and motivation to stay in a learning environment. Therefore, this result suggests that animated tutors are able to foster students' interest in the learning process and focus their attention on the most relevant information in a Web-based multimedia learning system.

The result of the preference survey shows that over $90 \%$ of the students state that they paid greater attention to the Web-based multimedia learning system. They also agree that it is more interesting, and they feel more at ease using it. Furthermore, the majority of the students (97\%) agree that the Web-based multimedia learning system increases their motivation and engagement in the learning environment. They also prefer to use it for independent learning after school.

\section{Conclusion}

This research is an extension of our previous project. It provides evidence from memory retention and comprehension tests by comparing a Web-based multimedia learning system with a Web-based PowerPoint learning system. The result of the post-test suggests that the Web-based multimedia learning system significantly improves students' learning and effectively teaches their problem-solving abilities. This increases the ability of students to apply their knowledge to solve traffic problems like those given in the problemsolving questions. However, the increase in comprehension is not necessarily accompanied by an increase in memory retention. The retention test of this experiment showed that in the Web-based multimedia learning system, students did not score significantly higher than in the PowerPoint-based learning system. The possible reasons for this may be:

- The animated agent integrated into our multimedia material may cause more efficient comprehension. Research by Lester and Stone [23] and Moreno et al. [24] has demonstrated that animated agents can generate important educational benefits by improving students' problem-solving abilities. They found that animated agents can provide a strong positive effect on students' learning experiences and by using speech, gaze and gestures, personalize the learning task and help students create a positive relationship with the virtual character. Therefore, when an animated agent is integrated in the multimedia environment, the computer increases the students' social response and promotes interest in the learning task. Such a phenomenon is also confirmed in our experiment.

In Table 2, Question $4(M=4.58, \mathrm{SD}=0.76)$ and $5(M=4.58, \mathrm{SD}=0.67)$ have high scores, revealing that students viewed the animated tutor like a mentor providing instructional knowledge and advice. Reeves and Nass [25] state that interactions between learners and virtual characters endowed with various social attributes create a kind of personal relationship. Animated characters can build and maintain long-term, socio-emotional relationships with their users [26]. Thus, the high scores reflect these theories and prove that animated tutors facilitate and enrich the interaction between student and computer in a learning context, and further help student with reducing the loads of understanding teaching materials.

Furthermore, Table 2 also shows that the scores from Question $1(M=4.68, \mathrm{SD}=0.60)$, Question $2(M=4.42$, $\mathrm{SD}=0.76)$ and Question $6(M=4.45, \mathrm{SD}=0.93)$ are higher than the average of 3 . This reveals that the animated tutor is really able to increase pupils' learning motivation and interest. In fact, existing studies find that an animated character can render a learning system more entertaining or engaging [3], [21]. Virtual characters make learning systems more life-like, and this increases students' motivation to stay in a learning environment. In our experiment, these motivated students rise in new problem-solving situations. Thus, we suggest that the results of effective learning are more obvious in problem-solving tests than in retention tests.

- Non-linear interactive structure is designed in our multimedia material so that it may foster deep learning. The Web-based multimedia learning system gives students the control panel and allows them to set their own learning pace. 
With the animated agent around the pane, students may feel that when they press a button in response to the animated agent, it presents new information according to their action. This may help students develop an active relationship with the material content. Mayer and Chandler [27] found the effect of learner interaction is able to reduce the students' cognitive load on their working memory. In their experiment, the interactivity took the form of a button which would reveal the next stage of the lesson when pressed. The students who used the interactive system had significantly higher transfer-test scores than those who used the noninteractive system. Evans and Gibbons [28] found a similar result in their study. They demonstrated that interactive systems can increase effective learning by engaging learners more closely with the material. They also suggested that adding interactivity to a computer-based lesson is able to increases learners' understanding.

- The animated agent's dialogues are highlighted on the screen so that it may reduce students' cognitive load. In our learning system, we not only provided the animated agent's advice to guide students as they process information, as well as reduce visual searching on the screen, but also highlighted its previous dialogues on the Tutor Dialogue section to help students organize the animated agent's explanations. Corresponding to Mayer's signaling principle [16], students learn more deeply when cues are added that highlight the main ideas and organization of the words. Thus, we thought such a highlight design may reduce extraneous information, and further promote a better comprehension test.

- The keywords of the material, 'Road Traffic Safety', are not very difficult to remember. One of the teachers in our experiment commented, "I think this material is common knowledge, and students easily associate the contents with their life." Thus, we think that students can easily recall the material after learning.

In addition, the previously presented questionnaire found pupils had more positive attitudes and interests when the lesson was taught by an animated tutor. The results of the attitude survey indicate that adding an animated tutor into a multimedia learning environment may foster students' learning activities and facilitate their engagement in the learning process. Furthermore, the majority of participating pupils think that the Web-based multimedia learning system was more interesting than the Web-based PowerPoint learning system. The results of the preference survey show that the pupils prefer to adopt the Web-based multimedia learning system to review school course work, and further engage in independent learning after school. Thus, this study may suggest that teachers in elementary education who adopt our tool can foster improved learning performance. We also hope that the Web-based multimedia learning system can play a scaffolding role to help pupils develop independent study skills after school.

However, the course, 'Road Traffic Safety', in this experiment is not a major course in Taiwan elementary education. In our future work, we will cooperate with teachers, students and their parents to design appropriate mate- rial for major courses, such as 'science', and further investigate the evidence of our research. Moreover, one of teachers in our experiment suggests, "If possible, more practical details or activities can be added to the Web-based multimedia learning system, such as on-line examinations or training, to improve the students' ability to use creative thinking and problem solving." Thus, we should develop a more powerful Web-based multimedia learning system in order to foster more effective learning in the future.

\section{Acknowledgments}

The authors would like to thank the National Science Council of R.O.C. under Contract No. NSC-97-2221-E-468-005 and Asia University under Contract No.97-I-06.

\section{References}

[1] R. Atkinson, R. Mayer, and M. Merrill, "Fostering social agency in multimedia learning: Examining the impact of an animated agent's voice," Contemporary Educational Psychology, vol.30, pp.117-139, 2005.

[2] Q. Dunsworth and R.K. Atkinson, "Fostering multimedia learning of science: Exploring the role of an animated agent's image," Computers \& Education, vol.49, pp.677-690, 2007.

[3] A. Gulz, "Benefits of virtual characters in computer based learning environments: Claims and evidence," Int. J. Artificial Intelligence in Education, vol.14, pp.313-334, 2004.

[4] E.M. Sims, "Reusable, lifelike virtual humans for mentoring and role-playing," Computers \& Education, vol.49, pp.75-92, 2007.

[5] W.L. Johnson and J.W. Rickel, "Animated pedagogical agents: Faceto-face interaction in interactive learning environments," Int. J. Artificial Intelligence in Education, vol.11, pp.47-78, 2000

[6] C.-I. Peña, J.-L. Marzo, and J.-L.D.L. Rosa, "Intelligent agents in a teaching and learning environment on the web," Proc. 2nd IEEE International Conference on Advanced Learning Technologies (ICALT2002), pp.21-27, 2002.

[7] M. Ueno, "Animated pedagogical agent based on decision tree for elearning," Proc. Fifth IEEE International Conference on Advanced Learning Technologies (ICALT'05), pp.188-192, 2005.

[8] T.-H. Chen, J.-M. Lin, K.-Y. Chin, and Z.-W. Hong, "Design of an IDML-based interactive agent drama authoring tool," Int. Conf. Intelligent Systems Design and Applications (ISDA2008), pp.67-72, Kaohsiung, Taiwan, 2008.

[9] K.-Y. Chin, J.-M. Lin, Z.-W. Hong, K.-T. Lin, and W.-T. Lee, "Developing an IDML-based embodied pedagogical agent system for multimedia learning," Ninth International Conference on Hybrid Intelligent Systems (HIS-2009), pp.35-41, Shenyang, China, 2009.

[10] Z.-W. Hong, K.-Y. Chin, and J.-M. Lin, "Developing embodied agent-based user interface by using interactive drama markup language," Third International Conference on Ubiquitous Information Management and Communication (ICUIMC-2009), Suwon, Korea, 2009.

[11] A. Savoy, R.W. Proctor, and G. Salvendy, "Information retention from PowerPoint and traditional lectures," Computers \& Education, vol.52, pp.858-867, 2009 .

[12] J.M. Apperson, E.L. Laws, and J.A. Scepansky, "An assessment of student preferences for PowerPoint presentation structure in undergraduate courses," Computers \& Education, vol.50, pp.148-153, 2008.

[13] J.E. Susskind, "Limits of PowerPoint's power: Enhancing students' self-efficacy and attitudes but not their behavior," Computers \& Education, vol.50, pp.1228-1239, 2008.

[14] A. Szabo and N. Hastings, "Using it in the undergraduate classroom: 
Should we replace the blackboard with PowerPoint?," Computers \& Education, vol.35, pp.175-187, 2000.

[15] W. Tsou, W. Wang, and H.-Y. Li, "How computers facilitate English foreign language learners acquire English abstract words," Computers \& Education, vol.39, no.4, pp.415-428, 2002.

[16] R.E. Mayer, The Cambridge handbook of multimedia learning, Cambridge University Press, New York, NY, 2005.

[17] R.E. Mayer, Multimedia learning, Cambridge University Press, New York, NY, 2001.

[18] M. Kobayashi, H. Sasaki, and M. Takeya, "Instructional navigation technology in a multimedia system for learner-centered learning," IEICE Trans. Inf. \& Syst., vol.E80-D, no.2, pp.189-195, Feb. 1997.

[19] J. Strobel, D.H. Jonassen, and I.G. Ionas, "The evolution of a collaborative authoring system for non-linear hypertext: A design-based research study," Computers \& Education, vol.51, pp.67-85, 2008.

[20] J. Cohen, Statistical power analysis for the behavioral sciences, 2nd ed., Erlbaum, Hillsdale, NJ, 1988.

[21] M. Moundridou and M. Virvou, "Evaluating the persona effect of an interface agent in a tutoring system," J. Computer Assisted Learning, vol.18, no.2, pp.253-261, 2002.

[22] K. Ariyasu, I. Yamada, H. Sumiyoshi, M. Shibata, and N. Yagi, "Visualization of text-based dialog in a virtual classroom for elearning," IEICE Trans. Inf. \& Syst., vol.E88-D, no.5, pp.836-842, May 2005.

[23] J.C. Lester and B.A. Stone, "Increasing believability in animated pedagogical agents," Proc. First International Conference on Autonomous Agents, pp.16-21, 1997.

[24] R. Moreno, R.E. Mayer, H.A. Spires, and J.C. Lester, "The case for social agency in computer-based teaching: Do students learn more deeply when they interact with animated pedagogical agents?," Cognition and Instruction, vol.19, no.2, pp.177-213, 2001.

[25] B. Reeves and C. Nass, The media equation: How people treat computers, televisions and new media like real people and places, Cambridge University Press, New York, NY, 1996.

[26] T. Bickmore, "Social dialogue is serious business," CHI 2002 Workshop on Socially Adept Technologies, 2002.

[27] R.E. Mayer and P. Chandler, "When learning is just a click away: Does simple user interaction foster deeper understanding of multimedia messages?,” J. Educational Psychology, vol.93, pp.806-813, 2001.

[28] C. Evans and N.J. Gibbons, "The interactivity effect in multimedia learning," Computers \& Education, vol.49, pp.1147-1160, 2007.

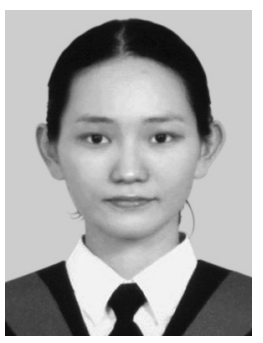

Kai-Yi Chin was born on July 21, 1978 in Nantou County, Taiwan. She received her M.S. degree in the Department of Information Science and Applications from Asia University, Taiwan, in 2003. She is currently a Ph.D. student at the Department of Information Engineering and Computer Science, Feng Chia University, Taichung, Taiwan. Her current researches include computer-aided learning and software agent.

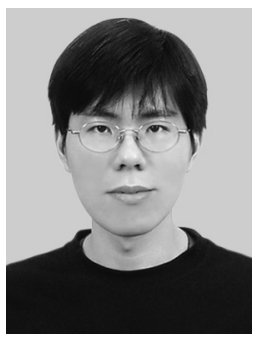

Yen-Lin Chen was born in Kaohsiung, Taiwan in 1978. He received the B.S. and Ph.D. degrees in electrical and control engineering from National Chiao Tung University, Hsinchu, Taiwan, in 2000 and 2006, respectively. He is now an Assistant Professor at the Dept. of Computer Science and Information Engineering, National Taipei University of Technology, Taipei, Taiwan. His research interests include image and video processing, pattern recognition, embedded systems, document image analysis, and intelligent transportation systems.

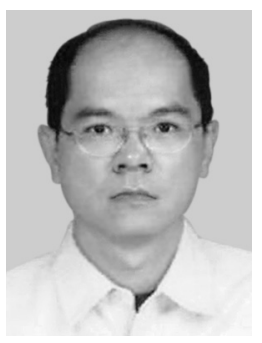

Jong-Shin Chen was born in 1972. He received the B.Sc. and Ph.D. degrees in computer science from Feng Chia University, Taiwan, in 1996 and 2003, respectively. Currently, he is an assistant professor in the Department of Information and Communication Engineering, ChaoYang University of Technology, Taiwan. His research interests include mobile computing, capacity planning, mobile agent, and wireless systems.

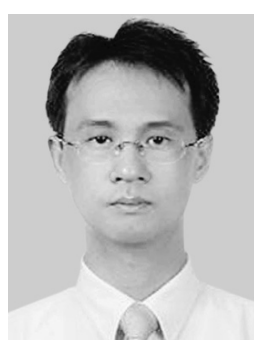

Zeng-Wei Hong was born on June 28, 1977 in Changhua, Taiwan. He received his M.S. and $\mathrm{Ph} . \mathrm{D}$. degrees in Information Engineering and Computer Science from Feng Chia University, Taiwan, in 2001 and 2006 respectively. He is currently an Assistant Professor at the Department of Computer Science and Information Engineering, Asia University, Taichung, Taiwan. His current researches include system reengineering, software agent, and scientific grid computing.

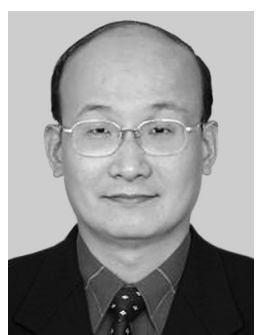

Jim-Min Lin was born on March 5, 1963 in Taipei, Taiwan. He received the B.S. degree in Engineering Science and the M.S. and the Ph.D. degrees in Electrical Engineering, all from National Cheng Kung University, Tainan, Taiwan, in 1985, 1987, and 1992, respectively. From February 1993 to July 2005, he was an Associate Professor at the Department of Information Engineering and Computer Science, Feng Chia University, Taichung City, Taiwan. Since August 2005, he has been a Full Professor at the same department. Since August 2009, he serves as the Chairman of the same department. Since November 2008, he also serves as the Secretary General of the Computer Society of the Republic of China (CSROC). His research interests include Operating Systems, Testable Design, Software Integration/Reuse, Embedded Systems, and Software Agent Technology. 